\title{
A.
}

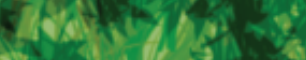

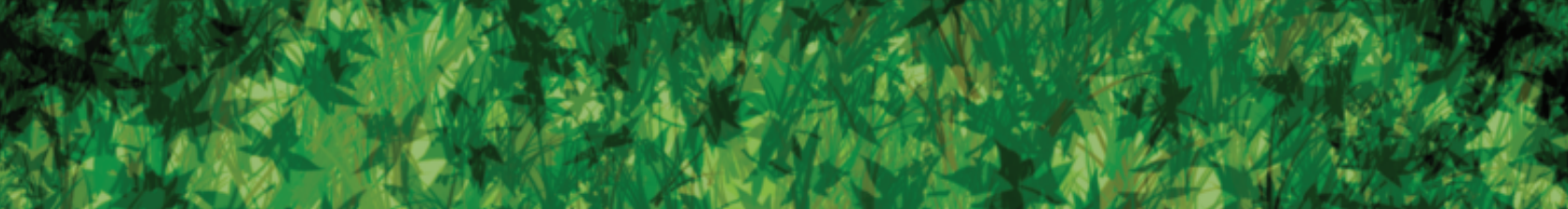

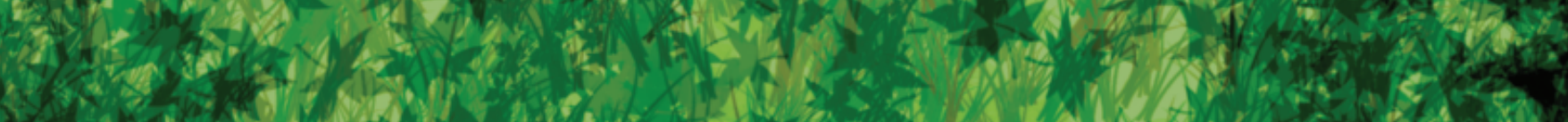

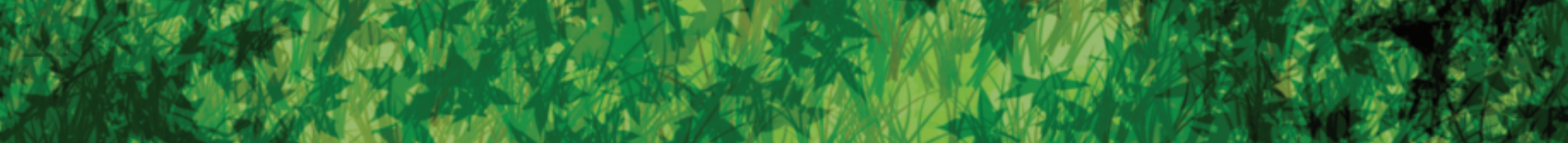

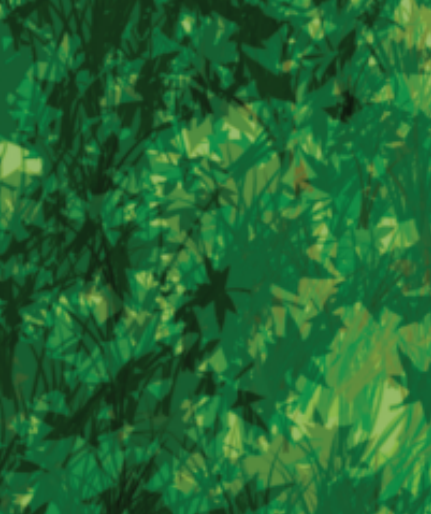

(4)

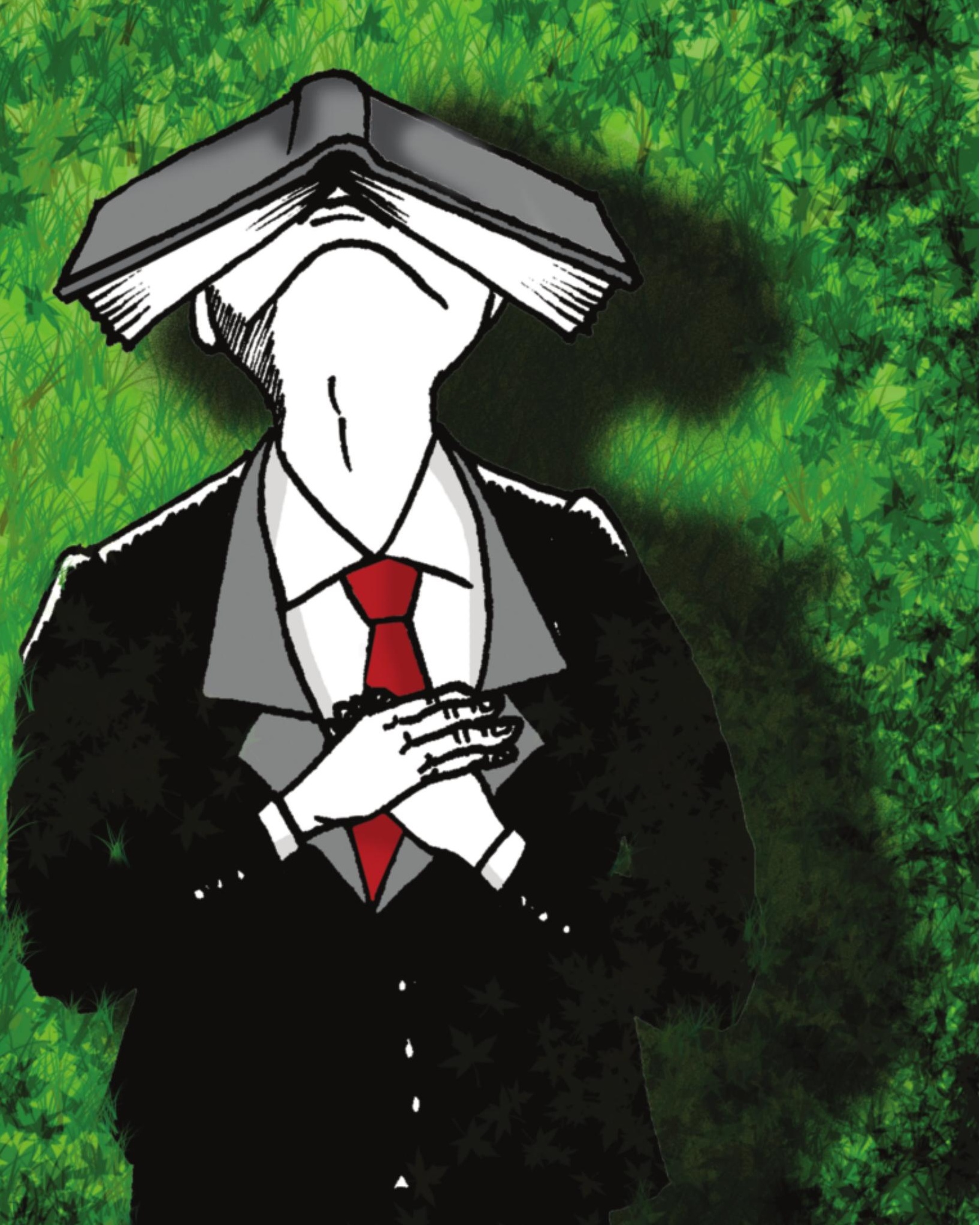

D.

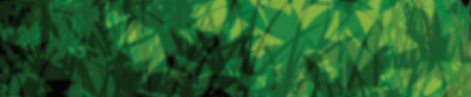

NF 


\section{Ciencia, educación \\ y poder}

\section{César Albornoz*}

\section{Una verdad de siglos}

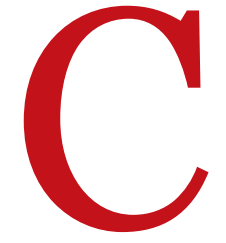

uando varios prestigiosos teóricos de las ciencias sociales de los últimos tiempos se refieren al desarrollo de la sociedad, teniendo como criterio predominante el aspecto tecnológico, hablan de al menos tres grandes momentos, o tres olas, como en el caso de Alvin Toffler: la primera correspondiente a la era agraria, la segunda a la era industrial y la tercera de la información o del conocimiento. ${ }^{1}$ Manuel Castells en su conocido libro La era de la Información también destaca las eras agraria, industrial y la que habría empezado desde fines de los años sesenta y primera mitad de los setenta del siglo pasado y que llama informacional. ${ }^{2}$ José de Souza Silva, siguiendo similar modelo diferencia cuatro paradigmas del emprendimiento social: extractivismo, agrarismo, industrialismo y el actual emergente que con dificultad denomina paradigma de intervención cuyo protagonista es el emprendedor social. ${ }^{3}$

* César Albornoz, M. A. en Sociología por la Universidad de Sofia Kliment Ojridski (Bulgaria) y máster en Comunicación Pública de la Ciencia y la Tecnología por la Universidad Central del Ecuador. Catedrático de la Facultad de Comunicación Social y de la Escuela de Sociología de la UCE. Coautor de: Centro histórico de Quito: Sociedad y Espacio Urbano (1990), Quito actual: Asentamientos populares (1992), El ferrocarril de Eloy Alfaro: El sueño de la integración (2008). Autor de: El pensamiento crítico ecuatoriano del siglo XX (1995), La corrupción y los gobernantes ecuatorianos en los primeros cien años de vida republicana (2000), Los grandes filósofos y la vida en el cosmos (2008). Ha publicado también artículos en varias revistas: Holograma y Textos y Contextos de la FACSO, en la Revista Ciencias Sociales de la Escuela de Sociología y en Anales Revista de la Universidad Central del Ecuador. Primer y tercer Premio Universidad Central del Ecuador en el Área Filosófica y Sociales en los años 2004 y 2007. Primer premio en el género de Ensayo, Ministerio de Cultura (2008). 
1 Alvin Toffler, La tercera ola, $7 \mathrm{ma}$ edición, Plaza \& Janes, Barcelona, 1981 2 Manuel Castells, La era de la información, Alianza Editorial, Madrid, 1998 3 José de Souza Silva, El Emprendimiento social en el cambio de época, Worlds \& Knowledges Otherwise, 2007 4 Peter Drucker, La sociedad post capitalista, 1974.

5 Edgar Morin, Los siete saberes necesarios para la educación del futuro, UNESCO/Santillana, Quito, 2003
Peter Drucker en la década de los setenta del siglo pasado introduce el concepto de sociedad del conocimiento,${ }^{4}$ noción que alcanza gran popularidad para caracterizar nuestra época. Simultáneamente varios sociólogos y economistas habían adoptado para la era que vivimos el apelativo de sociedad postindustrial (Daniel Bell, Galbrait, Touraine). Esquemas simplificadores de la realidad social que, sin embargo, han alcanzado amplia difusión por parte de las instituciones ideológicas del sistema imperante.

Punto coincidente en una amplia mayoría de autores es que en el siglo XXI se vive un verdadero cambio de época, en la que no hay posibilidad de desarrollo si no se avanza al ritmo que la ciencia y la tecnología imponen. Es decir, en la llamada sociedad del conocimiento, las políticas nacionales deberían tener como eje central de su desempeño, a la educación, tanto formal como informal de sus ciudadanos, orientada por nuevos paradigmas y quiebres epistemológicos. Uno de los más destacados propugnadores de ello es el pensador francés Edgar Morin, con apoyo y difusión de sus propuestas incluso por la UNESCO. ${ }^{5}$

Esa verdad, establecida ya por la modernidad desde al menos el siglo XVII (Bacon, Descartes, Leibniz, etc.) y generalizada por el movimiento de la Ilustración, antecedente ideológico de la fundación de los estados modernos que significó un cambio de época tan violento como el que actualmente vivimos, ha tropezado con obstáculos institucionales insuperables en muchos países como el nuestro. Pues, si se impusiera la racionalidad, no sería nada más fácil que adaptar modelos educacionales probados con éxito en el desarrollo de las sociedades del llamado primer mundo, o de sus experimentos en otras latitudes, como los de los famosos tigres del sudeste asiático, trillado ejemplo de la academia para decirnos que por ahí está una alternativa. Sociedades que hasta hace algunas décadas eran tan atrasadas como la mayoría del tercer mundo, que en poco tiempo han despegado con ritmos envidiables de crecimiento de las variables que maneja la macroeconomía.

\section{Añeja verdad en el Ecuador también}

Pero el funcionamiento de la sociedad es mucho más complejo y obedece a correlaciones de fuerzas expresadas en intrincadas relaciones de poder, que reflejan leyes objetivas del desarrollo social y, nos guste o no, ciencia y educación están subordinadas a coyunturas del poder político. Tal el caso ecuatoriano que, si indagamos en lo más avanzado del pensamiento nacional y en los planteamientos de sus más destacados exponentes, no han faltado propuestas y sin dificultad llegaríamos a establecer que desde los albores de la república, incluso antes con los precursores de la emancipación, ya se abogaba por una educación basada en los adelantos científicos y técnicos que viabilicen un desarrollo armónico de nuestra sociedad.

Eugenio Espejo, en el Nuevo Luciano de Quito, esboza temprana-

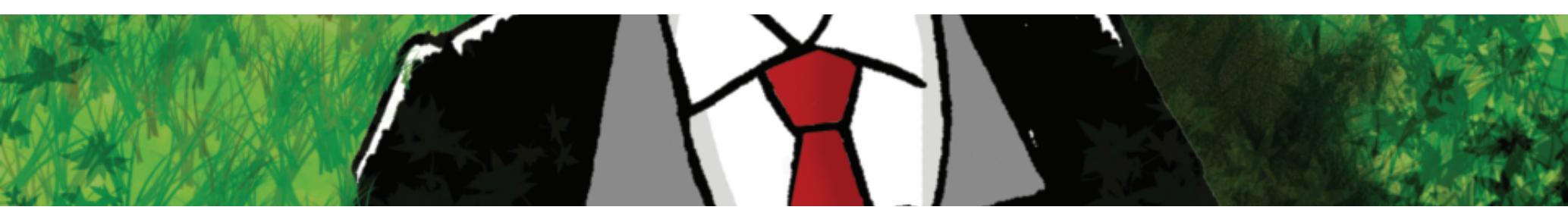


10 Luis Fernando Vivero, Lecciones de politica según los principios del sistema popular representativo, adoptado por la naciones americanas, Imprenta de Gaultier, París, 1827.

11 Alberto P. Cordero Aroca, Documentos políticos de la Administración de Don Vicente Rocafuerte Gobernador de la provincia de Guayaquil, t. I, Municipio de Guayaquil, Guayaquil, 2004, p. 69. 12 lbid., p. 72. 13 Ibíd., pp. 72-73.

14 Citado por Roberto Agramonte, La filosofía de Montalvo, t. I, Banco Central del Ecuador, Quito, 1992 p. 321.
Vivero, por ejemplo, en sus Lecciones de Política manifiesta que para salir del estado de postración y abatimiento que nos dejó el gobierno español, es necesario la más amplia educación para todo el pueblo, mujeres e indios incluidos: "Establecer una educación popular, mediante la cual todo el que nace en nuestro suelo, se ilustre, conozca su propia importancia y aprecie sus derechos". ${ }^{10}$

Como presidente del país, Rocafuerte, intenta poner las bases de una educación según el modelo liberal del cual era ideólogo y partidario. Mérito suyo es la fundación del primer colegio para señoritas en el país, de la Escuela de Obstetricia, del Instituto Agrario, de la Escuela Militar y Náutica, entre otras varias innovaciones. Más tarde, como gobernador de la provincia de Guayaquil, ante la ausencia de instituciones secundarias, funda el primer colegio en la ciudad porteña. En su discurso del 9 de octubre de 1841, dirigiéndose a los padres de familia de una escuela de niñas que visita, les dice que para superar el grado de retraso, "Una educación científica, aplicada a los usos y necesidades de la vida, es la que más conviene a países nuevos como los nuestros, en que es preciso poner en acción los elementos de riqueza que nos ofrece la variada fertilidad de nuestro suelo". ${ }^{11}$ Cuando el obispo de su ciudad reclama 3.142 pesos de réditos que causan los capitales impuestos sobre la Escuela Náutica, de prestigio continental y en la que se progresa en el estudio de ciencias exactas, su airada protesta, en carta al presidente Flores, no se hace esperar: "Yo he manifestado al obispo que estos fondos, siendo dedicados para la escuela náutica, es más útil para Guayaquil que mil seminarios, que por mucho tiempo, no serán más que planteles de ignorancia y de superstición". ${ }^{12}$

Ilusionado espera Rocafuerte el regreso de más de una decena de jóvenes guayaquileños enviados a París para formarse en ciencias, que, con nuevas ideas, impulsarían el desarrollo de la "agricultura, industria y comercio, levantando puentes y calzadas, dirigiendo caminos, abriendo canales, descuajando bosques, construyendo máquinas y buques a vapor y transformando por la magia de la ciencia, tristes desiertos en alegres poblaciones". ${ }^{13}$

Desgraciadamente para el Ecuador, este primer intento de reforma educativa y modernización no prospera por el predominio conservador que tomó el control del Estado desde 1860 hasta las postrimerías del siglo XIX.

Es tan deplorable el estado de la educación en el país que Montalvo, en 1880, en su célebre obra Las Catilinarias, protesta ante tanto retraso y oscurantismo y clama porque se enseñe ciencia: "Enseñadnos por Dios a leer y escribir, a contar y hacer cálculos: dadnos luces respecto de esta gran máquina del universo, qué cosa son los astros, de dónde nace la luz y lo que es el mundo mismo en que habitamos". ${ }^{14}$

Manuel J. Calle, en carta al presidente Alfredo Baquerizo Moreno fechada en Guayaquil el 28 de julio de 1915, da fehaciente testimonio de lo anterior:

“(...) los conservadores resultaron gerentes y gobernantes más ineptos, por cuanto bajo su gobierno de medio siglo no lograron sino adaptarse a formas educacionistas de régimen confesional, sin haber podido realizar un solo progreso digno de estimación (...) en cincuenta años no se propendió al desarrollo económico de la Nación, ni se construyó cosa de gran valer, sino dos carreteras inconclusas y costosísimas y un presidio y cuatro edificios que no importan lo que el atraso de la conciencia ecuatoriana y el anegamiento de la dignidad nacional en un mar fétido y cenagoso; que con ellos la República careció de crédito, la sociedad de nervio, el pensamiento de soberanía, la inteligencia de aire y de luz, en un

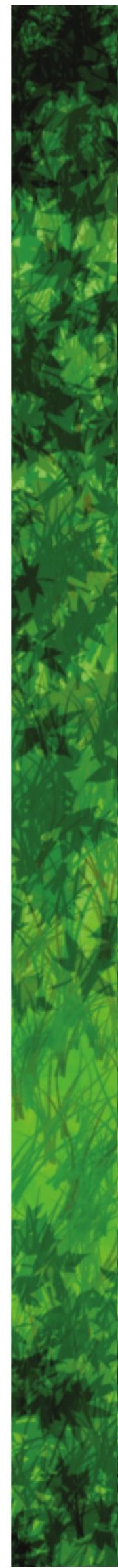


17 José Peralta, Informe del Ministro de Instrucción Pública al Congreso Ordinario de 1900, Imprenta de la Universidad Central, Quito, 1900, p. II. 18 Ver detalladamente esa oposición a la introducción del laicismo en Oswaldo Albornoz Peralta, Ecuador: luces y sombras del liberalismo, Editorial El Duende, Quito, 1989, p. 78 y ss. 19 Informe del Ministro de Instrucción Pública al Congreso Ordinario de 1900, op. cit., p. III. 20 Informe del Ministro de Instrucción Pública al Congreso Ordinario de 1901, Imprenta Nacional, Quito, 1901,

p. 5. 21 Ibid., p. 6.

22 René Báez, "El desembarco invisible. Ensayo sobre la funcionalización de las universidades ecuatorianas", en Reflexiones № 3, Revista de la Universidad Central del Ecuador, Quito, mayo de 2008, p. 14. todo destello de luz que pudiera penetrar en la oscurecida conciencia de las muchedumbres. Para tradicionalismo tan intransigente, no hay, no puede haber ciencia, fuera de los límites fijados por los intereses de secta; y toda mirada investigadora que se extienda más allá, es una ofensa a la Religión, un acto de rebeldía contra la Divinidad. ${ }^{17}$

Para muestra de la intolerancia y denodada resistencia a todo cambio de paradigmas, basta esta perla de lo que vociferaba la Iglesia, en oposición a la educación laica, a través de Federico González Suárez, uno de sus más preclaros representantes: "La educación laica es en lo moral tan contagioso como la elefancia: nadie puede vivir en contacto con el maestro laico y conservar sana el alma. En el orden moral, en el orden social y, sobre todo, en el orden religioso, la escuela laica es el culto de Moloch. El pecado que cometen los padres de familia consintiendo que sus hijos concurran a la escuela laica, no lo deja Dios impune". ${ }^{18}$

Ante el contubernio de los parlamentarios para impedir la reforma educativa propuesta por el liberalismo radical, Peralta les desenmascara:

Es inexplicable, Señores

Legisladores, que la Ley de

Instrucción Pública no haya destruido ya estos obstáculos, opuestos al adelantamiento del país por la intransigencia y el fanatismo más desatentados. ¿Cómo se quiere, pues, la libertad del pensamiento y la emancipación de la conciencia, la autonomía personal y la civilización verdadera, si la Ley no se atreve a romper de una vez esa cadena que nos ata a los tiempos de la Edad Media? ¿Cómo se quiere el triunfo de la Democracia y de la Libertad, si confiamos la educación de la juventud, esto es, la formación de los hombres del porvenir, a los mismos que han combatido y combaten sin tregua los principios liberales? ¿Cómo se quiere la ilustración de los pueblos, si encargamos la difusión de las luces a los mismos que luchan sin descanso por mantener el imperio de las tinieblas? ${ }^{19}$

Hasta el último momento de su gestión en la tarea que le encomendara el general Eloy Alfaro para llevar a cabo la reforma que demandaba el país, acortando distancias con los países más desarrollados, insiste ante los indolentes legisladores que, por su ideología y posición de clase, a una gran mayoría de ellos, lo que menos les interesa es el progreso $y$ bienestar de su pueblo: "repitoos, que conviene abrir nuevos campos a la actividad de la juventud ecuatoriana; puesto caso que la Jurisprudencia y la Medicina, únicas carreras profesionales hasta hoy, si útiles y nobles, no bastan para llenar las aspiraciones del país". ${ }^{20}$

Aplaude el restablecimiento de la Facultad de Ciencias Matemáticas en la Universidad Central y habla de la conveniencia de fundar, en el menor plazo posible, Institutos Agronómicos, Escuelas de Comercio, de Mecánica y de Química

Industriales, Veterinaria “y más profesiones prácticas y lucrosas, a fin de aprovechar todas las actividades y todas las aptitudes de la juventud y dar al mismo tiempo, un poderoso impulso al progreso de la república" ${ }^{21}$

Una plutocracia interesada en el control del Estado para hacer buenos negocios en el consolidado capitalismo mundial, a sangre y fuego frustró la posibilidad de que el Ecuador entonces alcance los niveles de productividad y competencia -como ahora se expresan los teóricos del liberalismo económico- a los que los liberales radicales quisieron llevarle en los albores del siglo XX. No le permitieron avanzar en su programa transformador a "un liberalismo humanista e integrador [que] jalonaba el futuro nacional con promisorias reformas políticas y económicas”, ${ }^{22}$ afirma René Báez.

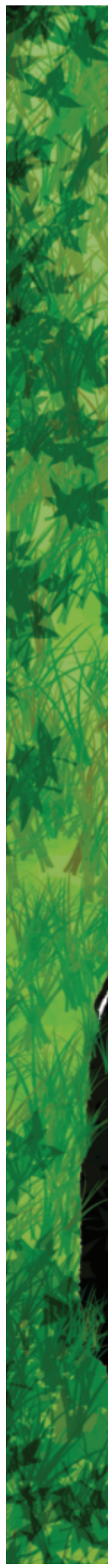


Sería largo historiar las propuestas truncadas durante todo el siglo anterior, por gobiernos y clases dominantes del país, entregados a proyectos y modelos de desarrollo inconvenientes para el interés nacional, convirtiendo al Estado en instrumento para la consecución de sus mezquinos fines. Tanto se ha escrito, para recordar solo uno de sus períodos más tenebrosos, sobre las últimas tres décadas de neoliberalismo devastador de nuestras economías, que si se les instaurara un juicio a todos los responsables, serían condenados a las más severas de las sanciones humanas. Basta recordar el estudio de Edgardo Lander sobre la ciencia neoliberal, ${ }^{23}$ documento por demás decidor, donde descubre cómo operan las transnacionales y corporaciones en la economía del mundo globalizado, con la anuencia de sus áulicos en nuestros países.

Y durante todos esos años siempre han habido voces y organizaciones sociales que han planteado el camino que deberíamos seguir como país para que Educación, Ciencia y Poder se articulen y coordinen acciones de manera que sirvan al desarrollo de nuestro pueblo. Pero la prepotencia de quienes se abanderaron de la libertad y del mercado, convirtiendo a Latinoamérica en la región de mayor injusticia social del planeta, fue la causante de la situación que hoy lamentamos, al despreciar e ignorar a estudiosos de nuestra realidad que señalaban el rumbo que el patriotismo aconsejaba. Nunca se ha hecho más patente que en nuestra historia común, esa verdad marxista que el capital no tiene patria.

Los más lúcidos analistas de nuestra realidad han insistido en ese único camino posible de desarrollo.

Uno de ellos es José Moncada, prestigioso economista y ex rector de la Universidad Central. Hace un cuarto de siglo ya definía lo que ha sido el quehacer científico dependiente de nuestro país: "La sociedad actual, la ciencia y la tecnología responden generalmente, a las orientaciones y conveniencias de quienes desde el hemisferio norte, nos dan modelos de consumo y de producción". Y en alianza estratégica "establecida entre los intereses foráneos y ciertos grupos minoritarios internos, en el Ecuador ha ido ganando terreno la idea de que lo verdaderamente importante, en materia científica y tecnológica, es cerrar las "brechas" que existen entre nuestro país y aquellos a los cuales se los ha exaltado a la categoría de países modelos". En ese marco, sigue su denuncia, "la investigación y la creación científica en el Ecuador" ha estado supeditada a donaciones de fundaciones y organismos internacionales vinculadas a los intereses y estilos de las "grandes corporaciones imperialistas y a la defensa y la preservación del actual sistema económico". Investigaciones e investigadores, entonces, "dedicados a recolectar datos y hacer análisis a ser utilizados como materia prima en el extranjero". ${ }^{24}$

Economía primaria de exportación y ciencia con iguales connotaciones. El neoliberalismo convirtió a un alto porcentaje de nuestros investigadores en sus empleados directos "a través de la contratación personal, del pago de subvenciones, de la selección de temas, de "sugerencias" de metodología, de entrega de bibliografía y de instrumental, de facilidades y financiamiento, de viajes a los centros metropolitanos de investigación". Así, "la ciencia y la tecnología se ha ido divorciando de la realidad nacional y enajenándose hasta el punto en el que, para muchos investigadores y técnicos ecuatorianos, carece de sentido toda lucha por la implantación de una autonomía científica que sólo podría darse si 

historia de los pueblos hispanoamericanos, Casa de la Cultura Ecuatoriana

Quito, 1988, p. 31 28 Ibíd., pp. 109, 115. es que se tiene como objetivo básico el cambio de la actual sociedad por otra de naturaleza socialista" ${ }^{25} \mathrm{Y}$ concluye en algo que ahora es un reto para quienes han enarbolado la bandera de lo que denominan socialismo del siglo $X X I$ :

En el marco de un proceso de cambio de la actual sociedad hacia un Ecuador socialista, debe darse prioridad a cierto tipo de tecnología no para cerrar "brechas", sino para satisfacer en mejor forma y lo más rápidamente posible las necesidades populares como vivienda, salud, alimentación, transporte colectivo, protección del ambiente, preservación de los recursos naturales no renovables, etc. ${ }^{26}$

Rodrigo Fierro, destacado científico y médico, expresa en una obra de hace dos décadas que, "a las puertas del siglo XXI, el de la revolución de la ciencia, en el que con toda seguridad se decidirá el futuro de los pueblos subalternos, entre los cuales se halla el mío, tan parcelado y tan disperso, me duele hasta la médula del alma nuestra situación de dependencia, en todos los campos y más en el científico y tecnológico, tan necesario actualmente para pretender ser libres" ${ }^{27}$

Y en larga cita, que merece ser recordada, hace la radiografía de la incuria de nuestros gobernantes y élites, como que hubiera sido escrita en nuestros días:

Con excepción de Cuba, no hay país iberoamericano que dedique más del $0.5 \%$ de su producto nacional bruto a su investigación científica y desarrollo tecnológico, en tanto que en algunos países desarrollados se acercan al 4\%. Esto resulta ser un buen indicador del subdesarrollo del pensamiento político en nuestras parcelas, pero ni aún procediendo con madurez, con racionalidad y sentido común, las patrias chicas podrían llegar a su independencia.

Los presupuestos del Instituto de Massachussets o de la Lomonosov son superiores al de muchos de los estados iberoamericanos juntos. La diferencia entre lo que invierte en ciencia y tecnología el país menos desarrollado entre los diez más desarrollados, es abismal si se compara con lo que invierte con igual propósito el menos subdesarrollado de los países del tercer mundo.

Los pueblos andinos, los países que conforman la región, ni aún integrados serían capaces actualmente de leer y escribir: de lograr su independencia. Tan solo unidos, todos los pueblos iberoamericanos, seremos capaces de hacer realidad "un hermoso sueño que fue el de Simón Bolívar: el pensar que algún día todo el continente latinoamericano pueda vivir con objetivos únicos bajo un solo gobierno federado" (...) Tan solo la integración nos permitirá crear un gran Instituto Tecnológico, en el que trabajaran con pasión los centenares de científicos iberoamericanos dispersos en el mundo, contar con un gran Centro de Información Científica y un banco de datos. Nos será dable dominar las técnicas más avanzadas y de esta manera iniciar nuestra aventura hacia la libertad..$^{28}$

En artículos más recientes vuelve a sus ideas de antaño, aconsejando a la mesa 7 de los asambleístas designados a promulgar leyes convenientes al desarrollo de la ciencia y de la tecnología nacionales en la nueva Constitución de Montecristi. Piensa que para paliar los males ocasionados por los países que en su imparable industrialización han deteriorado y deterioran la ecología del planeta, transgrediendo las más elementales normas del derecho internacional, culpables directos de una alarmante crisis alimentaria "con millones de muertos de hambre", es hora de prever nuestro futuro utilizando inteligentemente los bienes que podemos obtener de mar y tierra. Solo así, en su criterio, podremos remontar esos bajísimos niveles de producción por hectárea, dejando de usar semillas genéticamente debilitadas, fertilizantes y antiparasitarios inadecuados y empleo de tecnologías arcaicas. Un 
33 Elisa Sicouret, "Un libro necesario", Vistazo № 982, julio de 2008. 34 Arturo Andrés Roig en su obra El pensamiento social de Montalvo, refiere que la Sociedad de la Unión Americana de Santiago de Chile publica ya en 1862 una Colección de ensayos y documentos relativos a la unión y confederación de los pueblos hispanoamericanos, que compila muchas de las propuestas existentes para ese proyecto al que tendremos que llegar inevitablemente. investigación. No consideramos a la investigación como una prioridad y ésta se ve poco incentivada en el Ecuador". Confirma esa lacerante verdad por todos conocida, que apenas "alrededor del o,2 por ciento del presupuesto nacional se destina a investigación en el Ecuador, el menor porcentaje en Sudamérica”, dinero que se "canaliza a través de contadas instituciones, lo que hace aún más difícil su utilización para la mayoría de investigadores" y que "las universidades reciben escaso o nulo apoyo del Estado". ${ }^{33}$

\section{Abundan los estudios y propues- tas, falta voluntad política}

En América Latina ha sido una constante de casi dos siglos, si se recuerda a políticos y pensadores como Andrés Bello, Simón Rodríguez, Bolívar, Martí y una larga lista más, el desarrollo de corrientes de pensamiento con las más variadas denominaciones y vertientes ideológicas de lo que podría definirse, especialmente en nuestro tiempo, como pensamiento crítico.

Un denominador común para la gran mayoría de esos pensadores, en manifiesta tradición bolivariana también de vieja data, ${ }^{34}$ es la unidad latinoamericana, como mecanismo de integración que optimice el desarrollo regional, aprovechando factores históricos y culturales que harían de esa unidad casi un proceso natural. La Patria Grande para todo visionario latinoamericano sería nuestro único destino y garantía incluso de sobrevivencia, en un planeta en el que se estructuran bloques de poder regionales claramente definidos, convirtiéndolo en un campo de competencias sin oportunidad para sociedades pequeñas, económicamente débiles y sin control de las tecnologías de punta. Es decir, la conciencia de lo obvio es de conocimiento público y hasta habría unanimidad en amplios sectores de nuestras sociedades o patrias chicas, para constituirnos en esa próspera Unión
Latinoamericana, o al menos

Sudamericana en un inicio.

A pesar de esa ingente producción intelectual que traza rumbos por demás convenientes para construir sociedades más equitativas en nuestros respectivos países, en donde el desarrollo material sea garantía para elevar el nivel de vida de la gente y se erradiquen, $o$ al menos se minimicen los efectos de graves problemas sociales, evitando la agudización de la violencia social $u$ otras manifestaciones de una crisis generalizada, hay grupos sociales poderosos que se oponen con una tozudez digna de mejor causa.

Nuestros políticos, no solo por ignorancia sino por una moral al servicio de los más mezquinos intereses, convertidos en dóciles instrumentos de corporaciones, transnacionales, dueños del país y caciques locales, han impedido una y mil veces a lo largo de nuestra historia la concreción de esas iniciativas analizadas y reanalizadas, en lo mejor de la producción de nuestras ciencias sociales y en interminables investigaciones auspiciadas por las más diversas instituciones (Universidades, Ongs, fundaciones, organismos internacionales, etc., etc.).

La tragedia no es la inexistencia de diagnósticos, proyectos y programas, sino al contrario, la sobreabundancia de los mismos.

¿Qué es lo que realmente dificulta seguir un camino

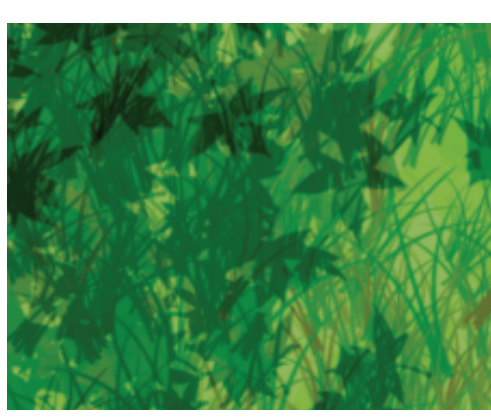
que la evidencia empírica demuestra ser beneficiosa en lo científico, técnico, económico, y el consiguiente mejoramiento de la calidad de vida, reconocimiento internacional, etc.? Indudablemente, factores que los sociólogos llaman exógenos y endógenos, o dichos en términos políticos más pertinentes colonialismo o neocolonialismo.

Cuando un país ha optado por un programa dependiente de su existencia 
por la subordinación económica, política y cultural respecto de ciertas metrópolis la famosa teoría del centro y la periferia-, está condenado irremediablemente a mantenerse en el retraso con todas sus consecuencias. Pero los culpables de esa opción tienen nombres y apellidos: esos enemigos internos del progreso y bienestar social, desde los gobernantes que por décadas subordinaron su gestión a designios foráneos opuestos al interés de los pueblos que confiaron en sus ofertas demagógicas para llegar a controlar la política, hasta los funcionarios, asesores y los investigadores sociales que con su producción justificaron el rumbo impuesto por los poderes extranjeros; en fin, toda esa cohorte que vistió y viste traje de librea, por unas cuantas monedas en que tasaron, desde organismos internacionales, el precio de su inmoral comodidad.

En el caso ecuatoriano, la consolidación de un Estado oligárquico terrateniente a lo largo de todo el siglo XIX y, luego, el de una burguesía retrógrada compradora y vendedora, que en lugar de tomar el rumbo industrial planteado por la revolución liderada por Alfaro -a la que, en contubernio con los conservadores y los intereses de las potencias foráneas, le prendieron fuego en la piras de El Ejido en enero de 1912-, son los responsables institucionales directos del atraso de nuestro país, al impedir que se enrumbe por la vía de desarrollo pregonado por las mentes más lúcidas de la patria.

Desde su posición de clase antinacional y desde su ignorancia e indiferencia hacia las nuevas tendencias del mundo al que viajaban y viajan en giras turísticas, a despilfarrar la riqueza nacional, producto de las ganancias obtenidas de la economía primaria, que nos ha convertido en proveedores de materia prima del primer mundo, fueron cómplices interesados de ese oprobioso neocolonialismo. Tan antinacional ha sido y es esa abrumadora mayoría de empresarios, que incluso invierten sus mal habidos capitales en el exterior, en lugar de dinamizar la economía nacional. Por esa actitud han llegado a su plena decadencia y en muchos lugares de América Latina despierta un pueblo que quiere recuperar la soberanía conculcada y tomar las riendas de su destino con un proyecto de desarrollo propio que haga realidad esa segunda independencia pregonada por José Martí.

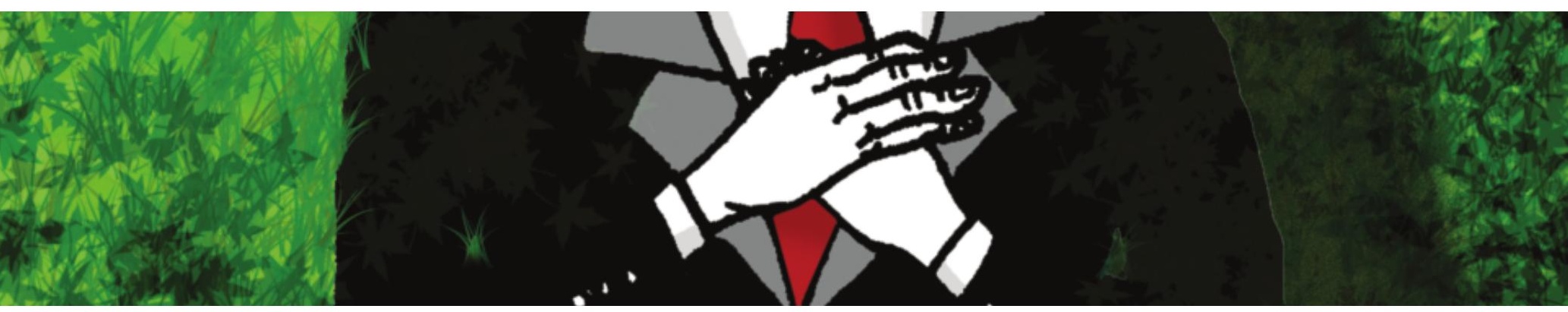

\section{¿Al fin enmendaremos la secular estupidez política?}

En los momentos actuales surgen las interrogantes de que si habrá sido necesario pasar ese tortuoso proceso de ineptitud política, entreguismo a intereses antinacionales e incuria ante una inevitable integración regional y, por fin, después de casi dos siglos de existencia y veinte constituciones, estaremos a las puertas de poner las bases de un desarrollo auténticamente nuestro, en el que se privilegie al ser humano, al ciudadano que sería el nuevo sujeto de la historia.

En referéndum realizado el 28 de septiembre del 2008 el pueblo ecuatoriano aprobó una nueva Constitución, en cuyo Art. 25 se indica que "Las personas tienen 
45 César Paz y Miño, "Quo vadis ciencia ecuatoriana (III)", El Telégrafo, Guayaquil, 10 de Julio del 2011
En todo caso, las expectativas son grandes. Por primera vez, en un siglo, después de los proyectos de la Revolución Alfarista se retoma el camino trazado por sus más connotados líderes. Quedamos a la espera de ver cómo de las palabras, planes, proyectos y metas se pasará a los hechos, cómo se institucionalizan y se afinan detalles de leyes que pueden aparecer innovadoras, pero al rato de su puesta en marcha, los resultados no sean tan halagüeños. Ojalá Ciencia, Educación y Poder se integren y se coordinen acciones, y sea cierto para el pueblo ecuatoriano eso de que La Patria ya es de todos, consigna que de no concretarse se devaluará como tantas otras que sembraron ilusiones e incumplieron, convirtiéndose en la más perversa de las demagogias.

\section{Bibliografía}

- Agramonte, Roberto, La filosofía de Montalvo, t. I, Banco Central del Ecuador, Quito, 1992.

- Albornoz Peralta, Oswaldo, Ecuador: luces y sombras del liberalismo, Editorial El Duende, Quito, 1989.

- Arroyo, María Belén, "Ecuador no le apuesta a la ciencia”, Entrevista a Luis Romo Saltos en El Universo, Guayaquil, 6 de abril de 2003.

- Báez, René, "El desembarco invisible. Ensayo sobre la funcionalización de las universidades ecuatorianas", en Reflexiones $\mathrm{N}^{0} 3$, Revista de la Universidad Central del Ecuador, Quito, mayo de 2008.

- Calle, Manuel J., Epistolario, Banco Central del Ecuador, Cuenca, 1989.

- Castells, Manuel, La era de la información, Alianza Editorial, Madrid, 1998.

- De Souza Silva, José, El Emprendimiento social en el cambio de época, Worlds \& Knowledges Otherwise, 2007.

- Cordero Aroca, Alberto P., Documentos políticos de la Administración de Don Vicente Rocafuerte Gobernador de la provincia de Guayaquil, t. I, Municipio de Guayaquil, Guayaquil, 2004.

- Drucker, Peter, La sociedad post-capitalista, 1974.

- Espejo, Eugenio, Obras completas, t. III, Casa de la Cultura Ecuatoriana Benjamín Carrión, Quito, 2008.

- Fierro, Rodrigo, La escritura en la historia de los pueblos hispanoamericanos, Casa de la Cultura Ecuatoriana, Quito, 1988.

- - - - - "Régimen de desarrollo", El Comercio, Quito, 5 de junio de 2008.

- - -_-_ "Señores de la Mesa 7", El Comercio, Quito, 12 de junio de 2008.

- - -_-_ "Bien por el Gobierno", El Comercio, Quito, 4 de agosto de 2011.

- Imbaquingo, Olga, "Hacer ciencia es una quimera", El Comercio, Quito, 19 de enero de 1992.

- Lander, Edgardo, La ciencia neoliberal, Fundación Venezolana de Promoción del Investigador, Caracas, 2005.

- Moncada Sánchez, José, Evolución y situación actual del capitalismo ecuatoriano y perspectivas de desarrollo socialista, Facultad de Economía de la Universidad Central, Quito, 1982.

- Morin, Edgar, Los siete saberes necesarios para la educación del futuro, UNESCO/Santillana, Quito, 2003.

- Paladines, Carlos (ed.), Juicio a Eugenio Espejo, Casa de la Cultura Ecuatoriana Benjamín Carrión, Quito, 2007. 
- Paz y Miño, César “Quo vadis ciencia ecuatoriana (III)”, El Telégrafo, Guayaquil, 10 de Julio del 2011

- Peralta, José, Informe del Ministro de Instrucción Pública al Congreso Ordinario de 1899, Imprenta de la Universidad Central, Quito, 1899.

- -_-_-_-- Informe del Ministro de Instrucción Pública al Congreso Ordinario de 1900, Imprenta de la Universidad Central, Quito, 1900.

- - -_-_---, Informe del Ministro de Instrucción Pública al Congreso Ordinario de 1901, Imprenta Nacional, Quito, 1901.

- República del Ecuador, Nueva Constitución del Ecuador, Publicación de la Universidad Central del Ecuador, Editorial Universitaria, Quito, 2008.

- Roig, Arturo Andrés, El pensamiento social de Montalvo, Editorial Tercer Mundo, Quito, 1984.

- Sicouret, Elisa, “Un libro necesario", Vistazo No 982, julio de 2008.

- Toffler, Alvin, La tercera ola, séptima edición, Plaza \& Janes, Barcelona, 1981.

- Vivero, Luis Fernando, Lecciones de política según los principios del sistema popular representativo, adoptado por la naciones americanas, Imprenta de Gaultier, París, 1827. 